\title{
MACROECONOMICS DURING PANDEMIC: CONSUMER BEHAVIOUR AND OPTIMAL CONTAINMENT POLICY
}

\section{TSOTNE MARGHIA}

\author{
PhD Student,
}

Ivane Javakhishvili Tbilisi State University, Georgia

t.marghia@mof.ge

Abstract. The COVID-19 pandemic has led us to the need of choosing between short-term and long-term priorities. In order to conduct and evaluate effective policies, new types of models are required, that are able to incorporate both epidemiological and economic variables. The aim of this paper is to make practical use of theoretical framework in case of Georgia and lay the foundation for future research. The theoretical framework of the paper is based on the macroeconomically extended version of epidemiological SIR type model developed by Eichenbaum et al. (Eichenbaum, Rebelo, Trabandt, 2020). In the homogenous economic agent model human behavior determines the epidemiological situation in the country. Individuals are reducing the probability of infecting themselves by reducing consumption and labor supply. However, consumers do not take into account the possibility of them infecting others, thus creating the negative external effect. Therefore, these effects can be internalized using the containment policy, which is represented as tax on consumption in the model. By raising taxes, the government increases the relative price of consumption to leisure (the economic agent chooses what part of one's time to devote to the labor market and what part of time to devote to leisure). Consequently, on other equal terms, households will change their behavior and prefer to stay at home, which is their response to the increased tax. However, these actions have a big impact on output. An epidemic ends when a large part of the population acquires collective immunity. In the absence of a vaccine and effective treatment, immunity can be generated by transmission of the virus and subsequent recovery. At this time, the number of deaths due to the virus is increasing. In this model, it is optimal to gradually increase the containment measures in parallel with the increase in virus cases, and to gradually mitigate the measures as the community approaches the critical level of immunity. This will help increase the share of people with immunity and, in the event of a high level of infection, deter infected people.

The paper uses the mentioned theoretical framework in practice, taking into account the epidemiological and economic state in Georgia and investigates the short and long run effects of the pandemic on population health, as well as their economic behavior. In the short run the containment policy leads to deep recession, although it reduces the number of infected and deceased. Therefore, in this case there is a choice to be made between epidemiological and economic situations in the short run. However, in the long run, the negative impact of the pandemic on the economy is weaker when using the containment policy, than in case of not using it at all.

KEYWORDS: COVID-19, CONTAINMENT POLICY, SIR MACRO MODEL, EPIDEMIC, RECESSION.

For citation: Marghia, T., (2020). Macroeconomics During Pandemic: Consumer Behavior and Optimal Containment Policy. Globalization and Business, 10. 103-114. https://doi.org/10.35945/gb.2020.10.013 


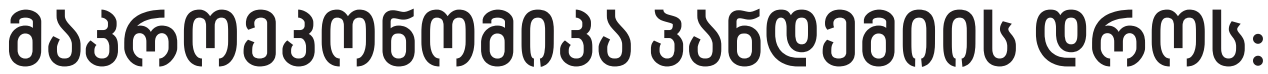

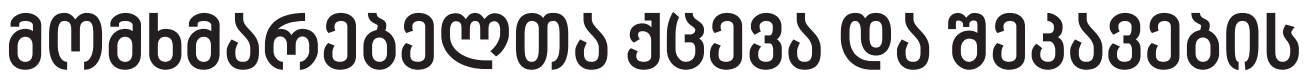

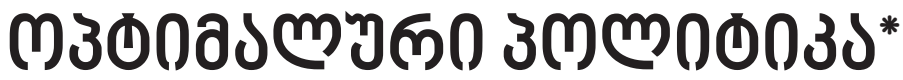

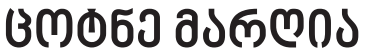

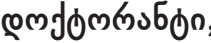

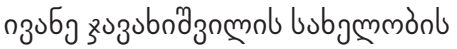

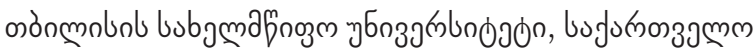

t.marghia@mof.ge

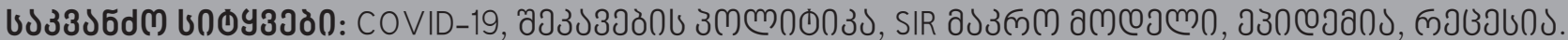

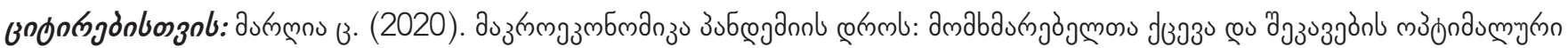

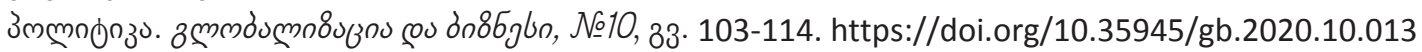

\section{1. gybuzumo}

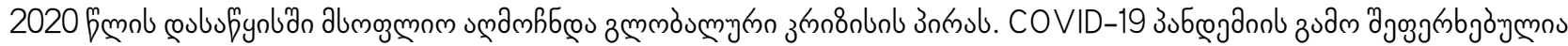

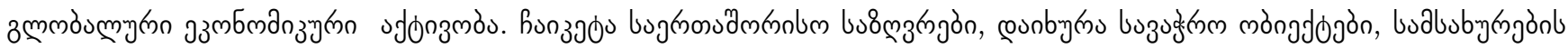

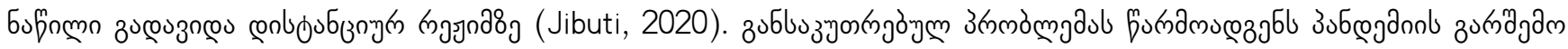

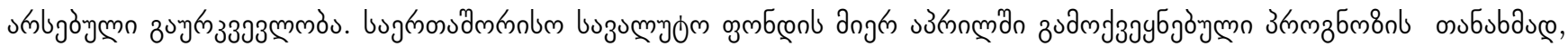

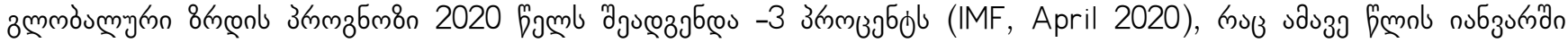
зง

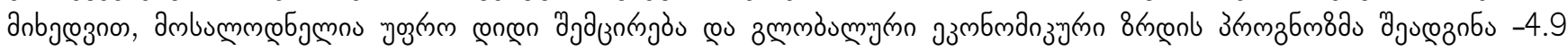

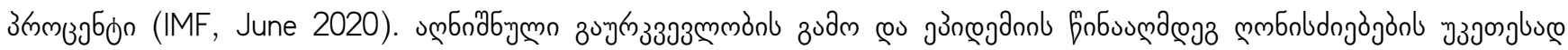

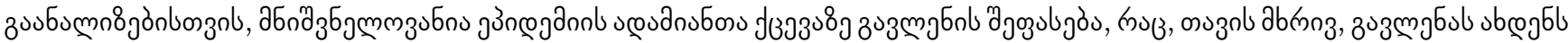

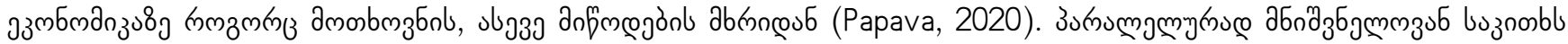

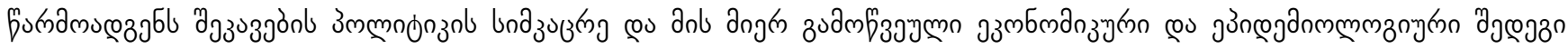

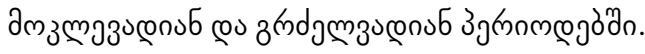

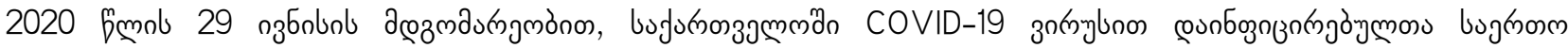

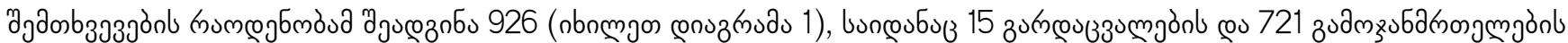

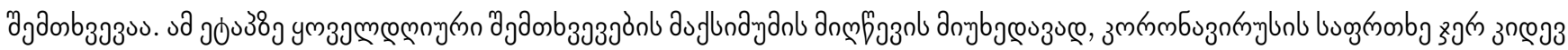

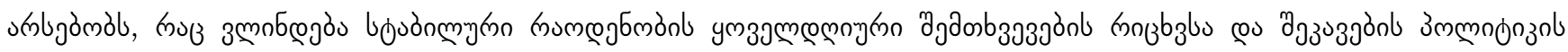

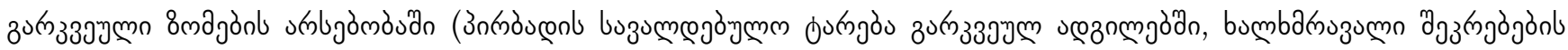

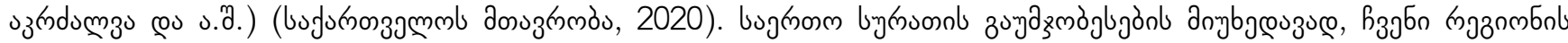

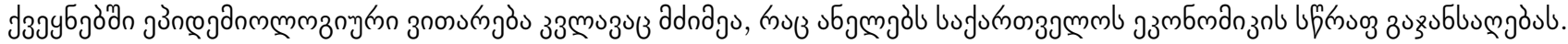

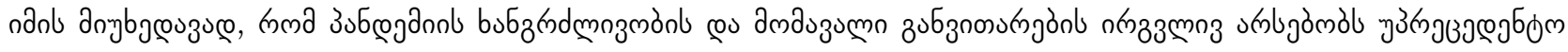

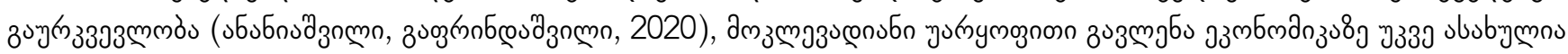

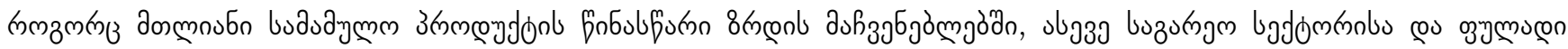

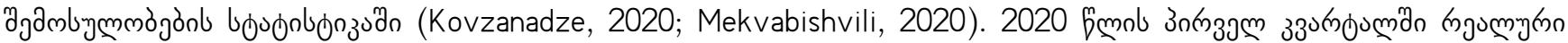

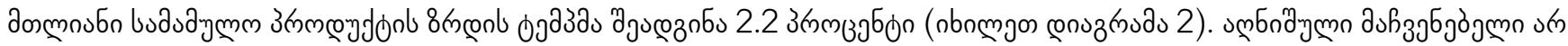

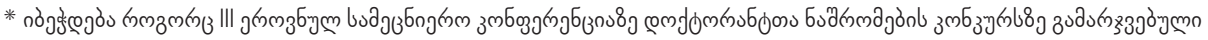




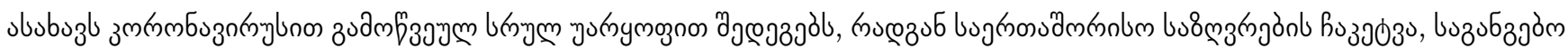
a

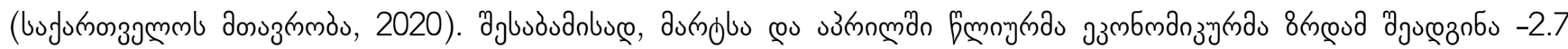

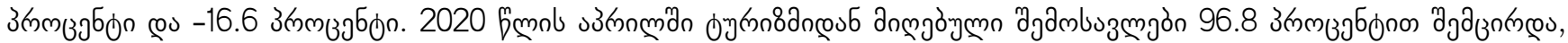

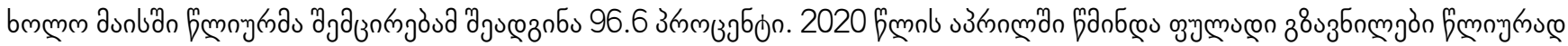

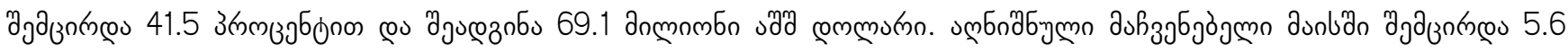

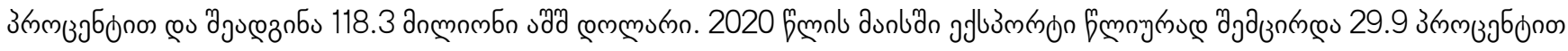

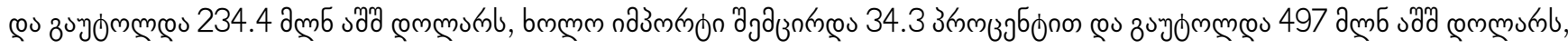

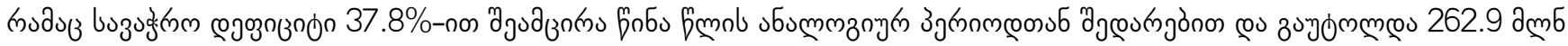

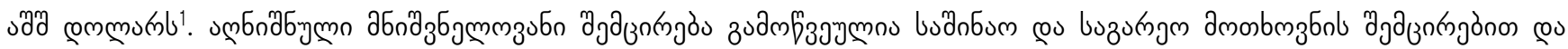

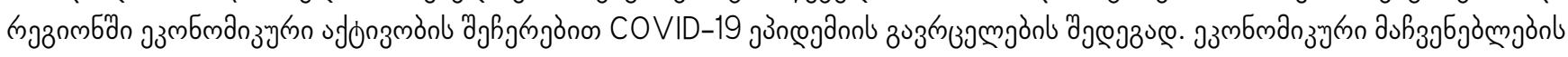

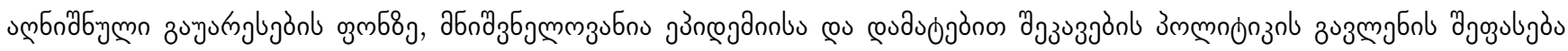
эзмбмдапзовे.

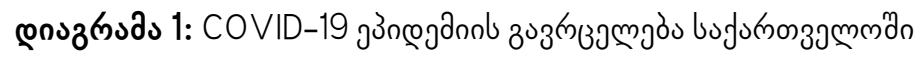
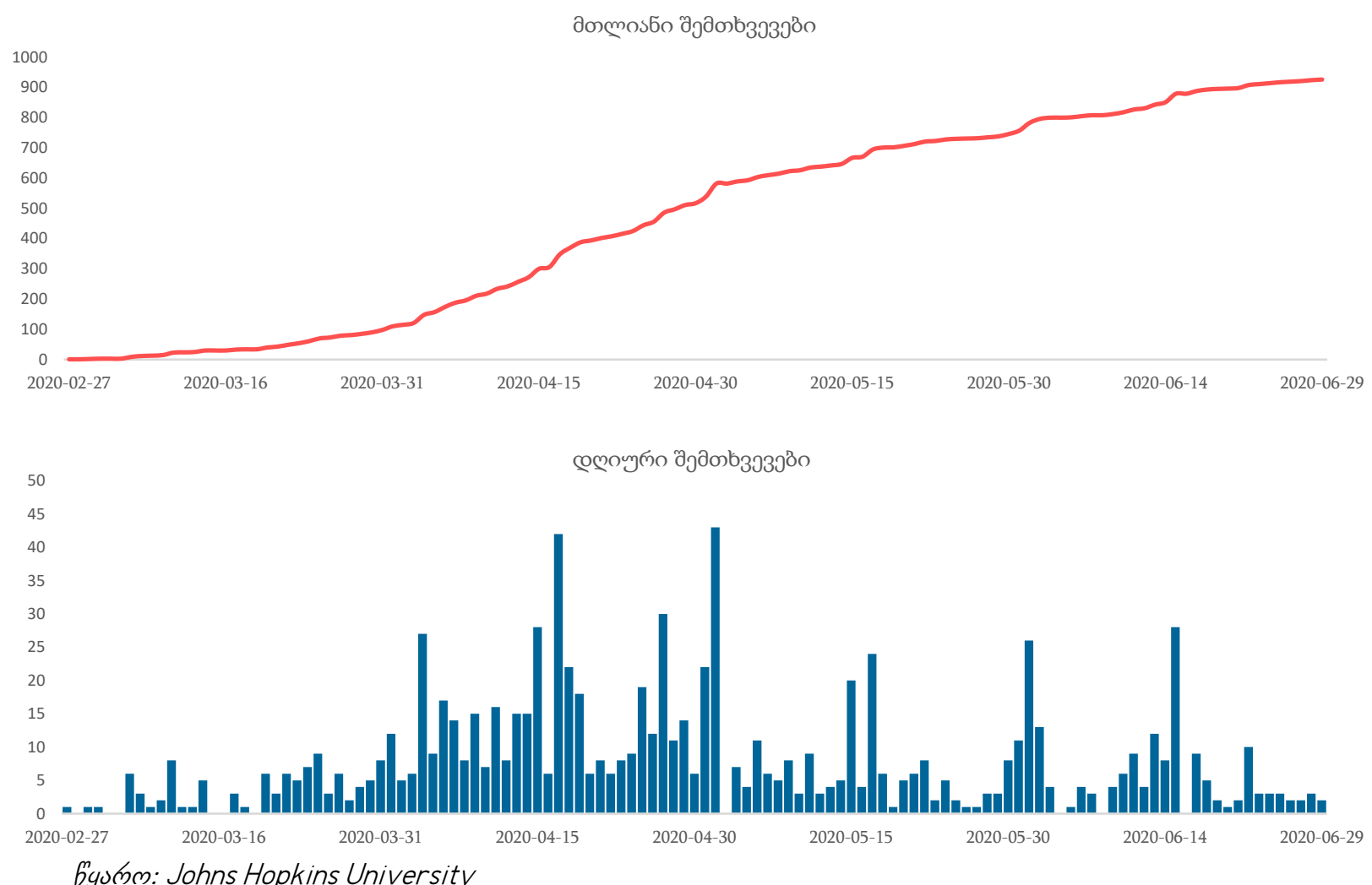

fyurn: Johns Hopkins University

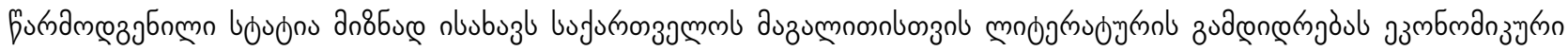

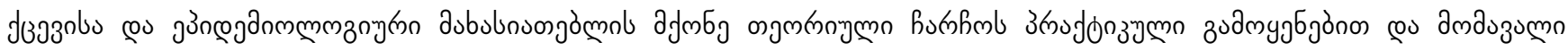

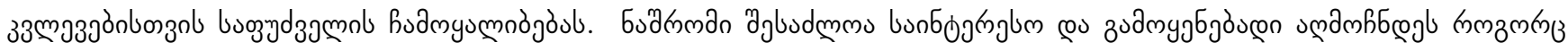

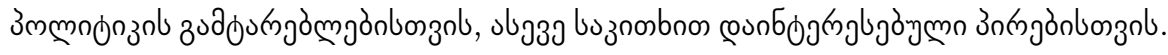

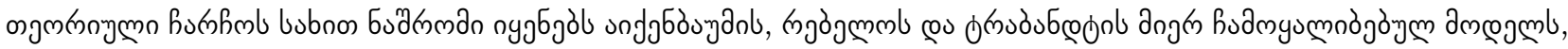

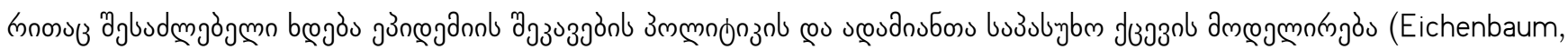

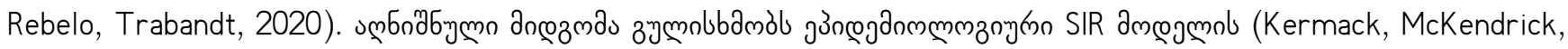

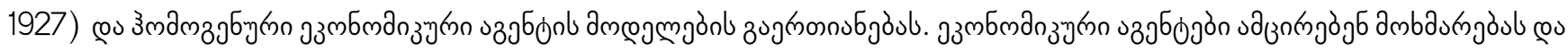

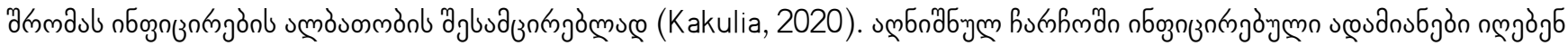

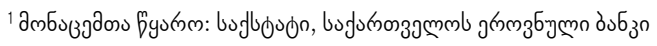




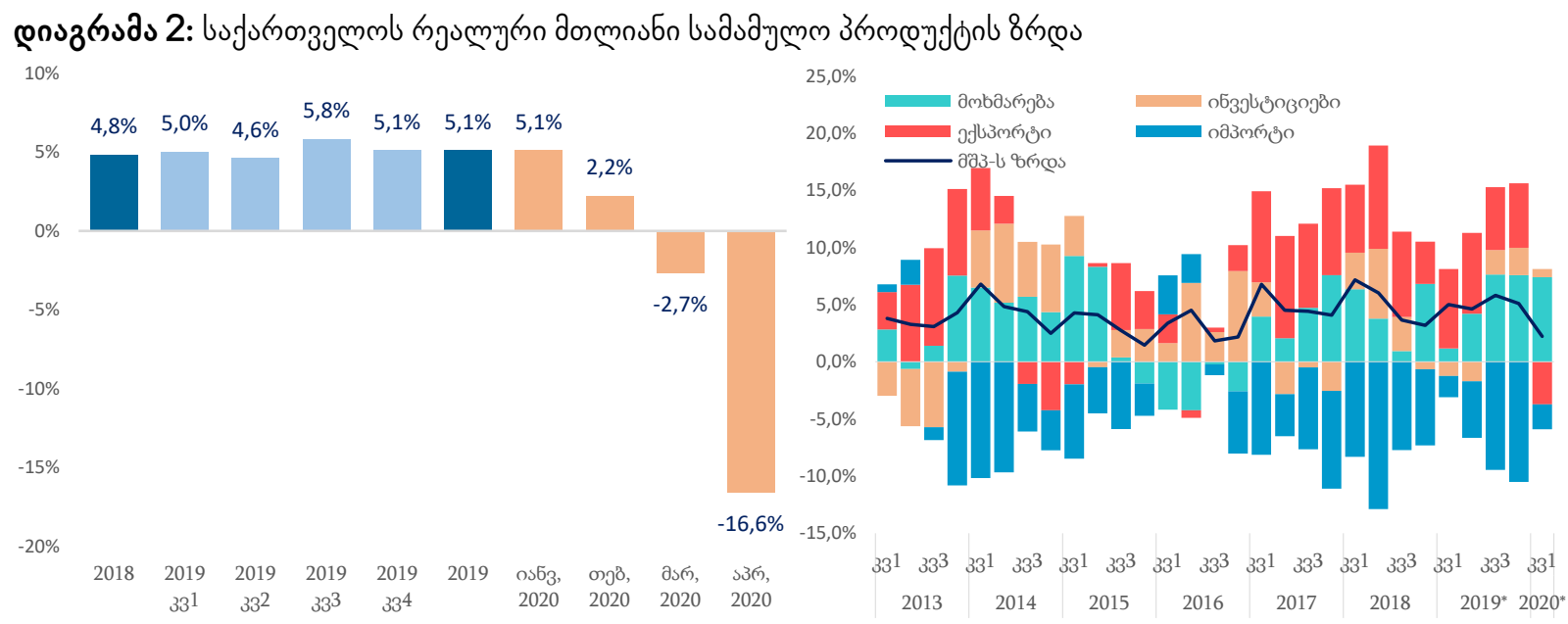

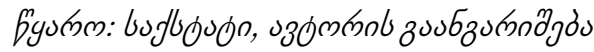

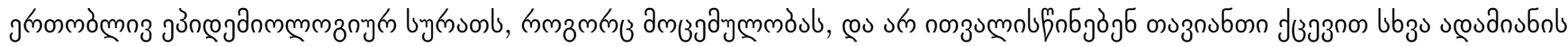

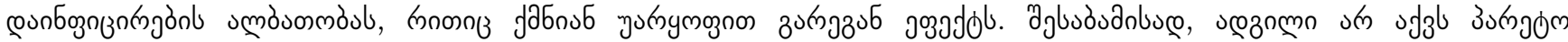

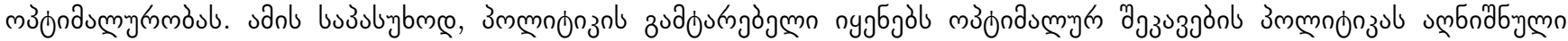

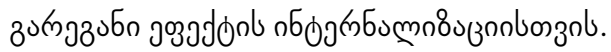

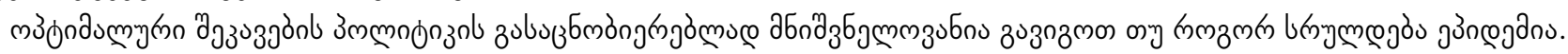

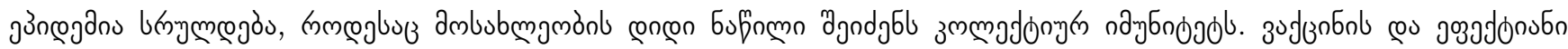

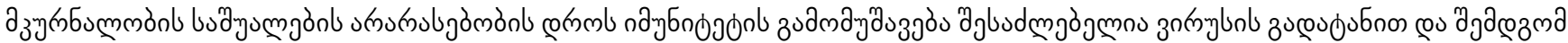

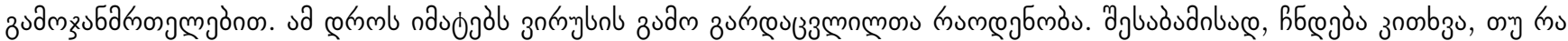

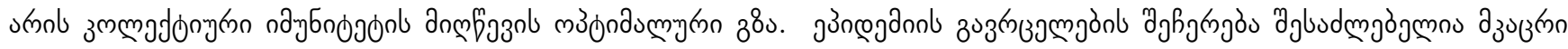

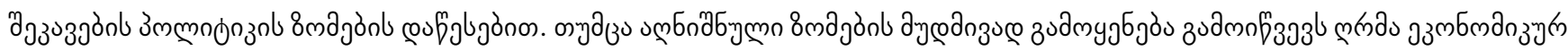

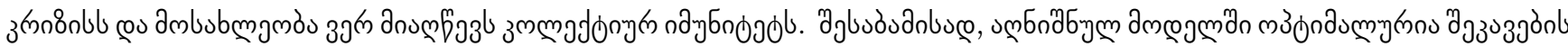

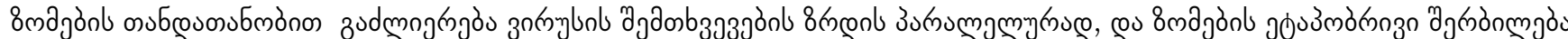

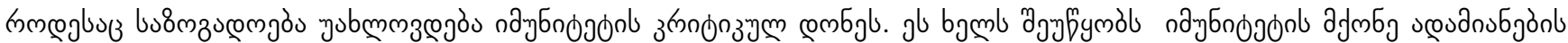

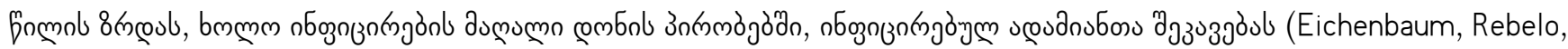
Trabandt, 2020).

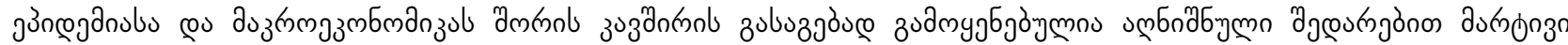

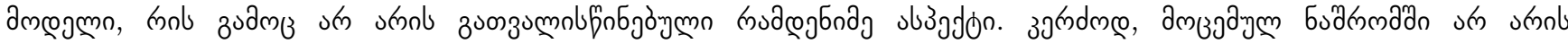
zumzumn

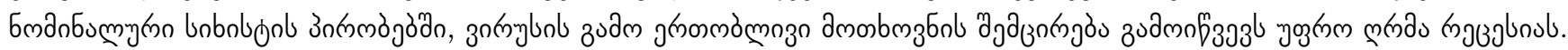

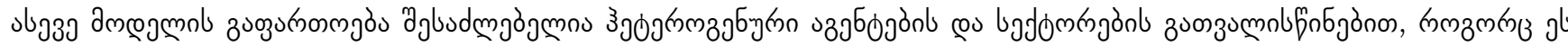

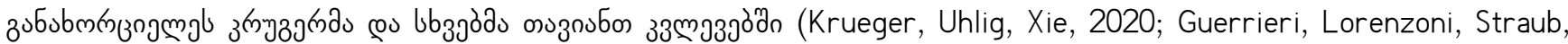

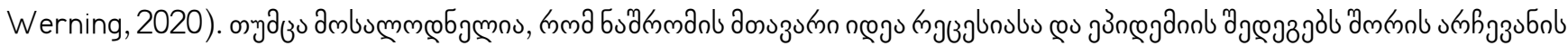

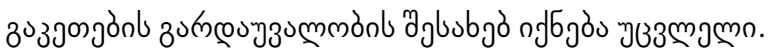

\section{2. modjmodynol andmbngmzs}

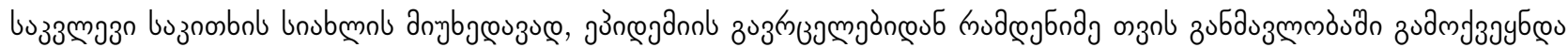

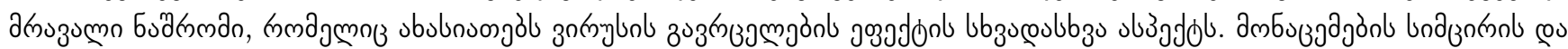

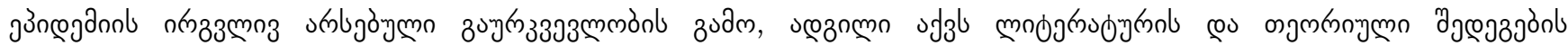

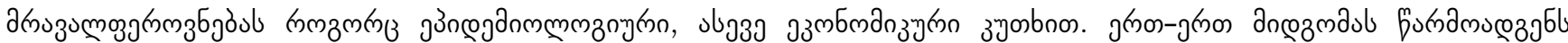

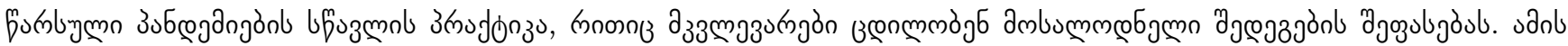

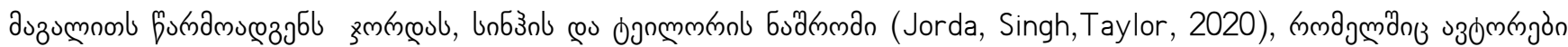

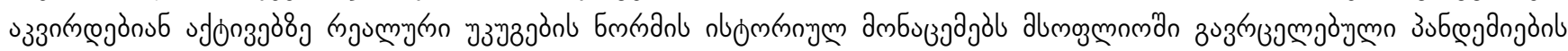




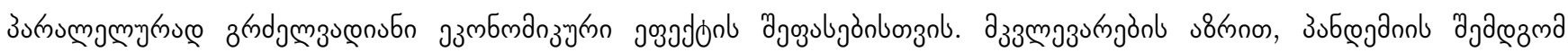

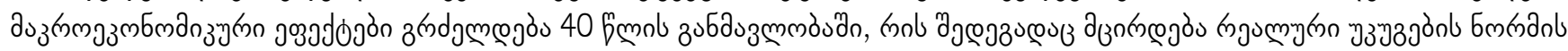

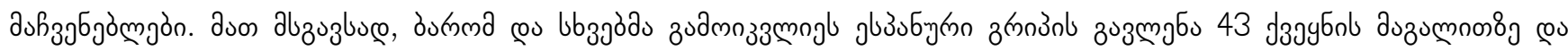

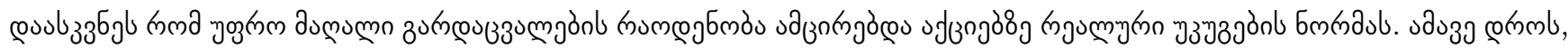
3 (Barro, Ursúa, Weng, 2020).

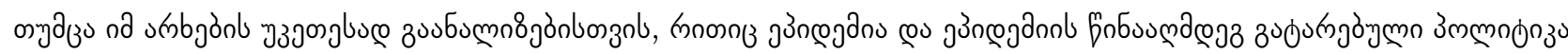

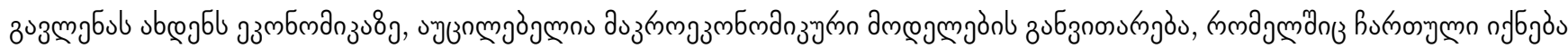

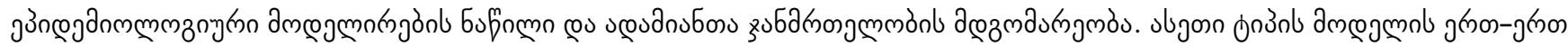

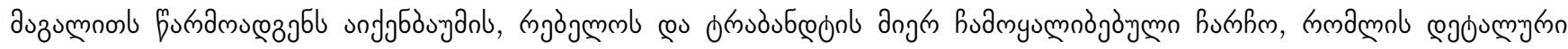

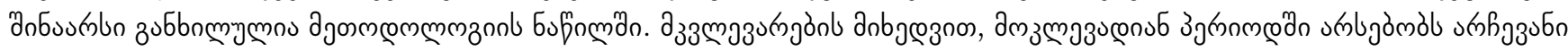

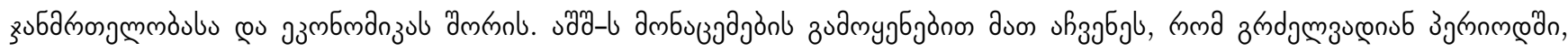

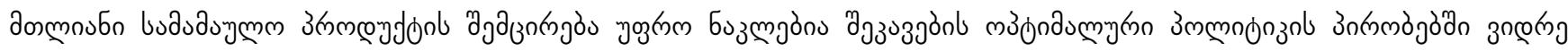

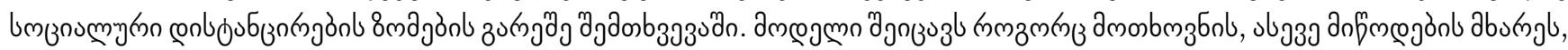

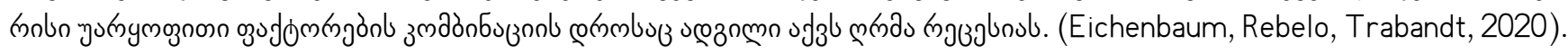

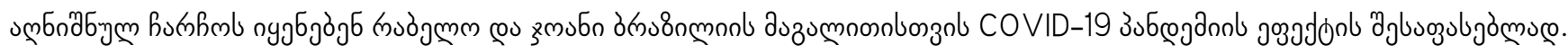

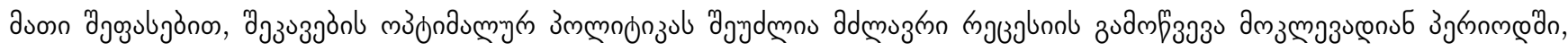

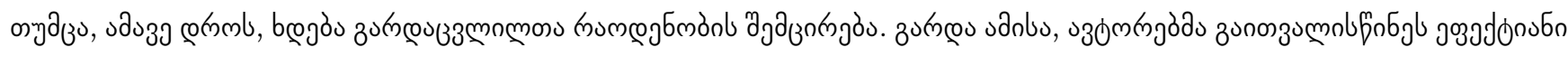

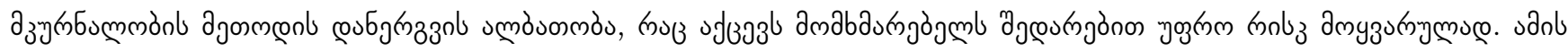

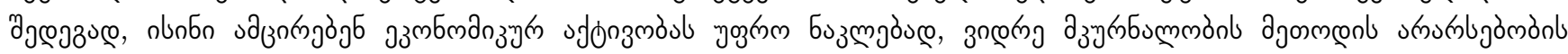

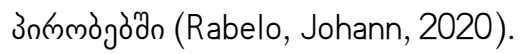

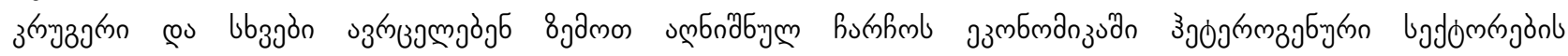

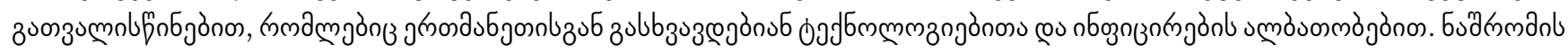

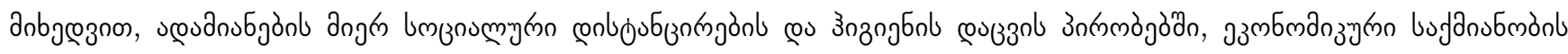

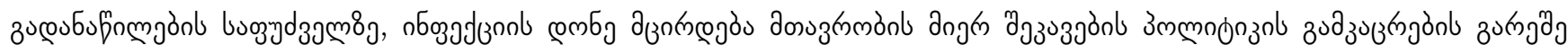

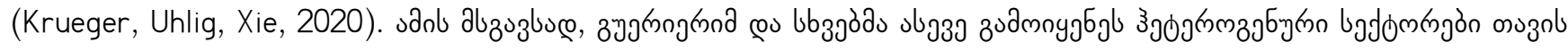

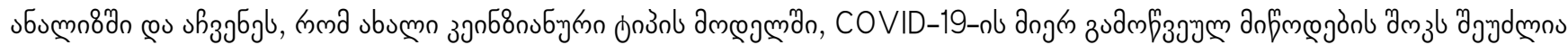

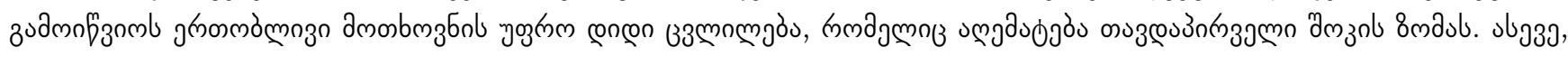

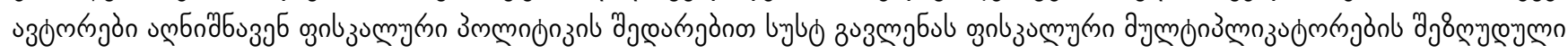
jơjJdon zudm (Guerrieri, Lorenzoni, Straub, Werning, 2020).

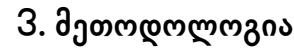

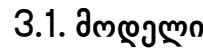

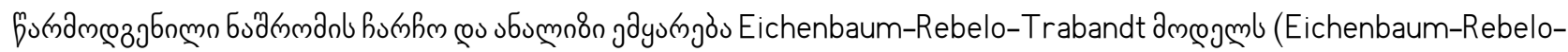

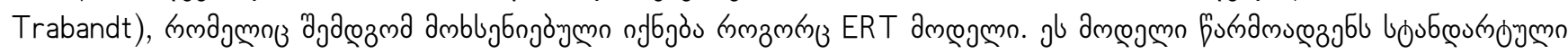

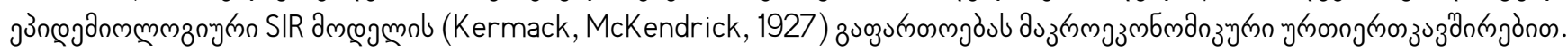

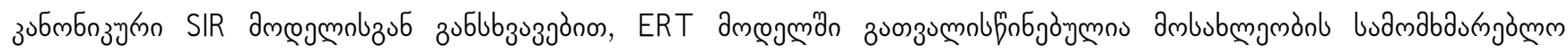

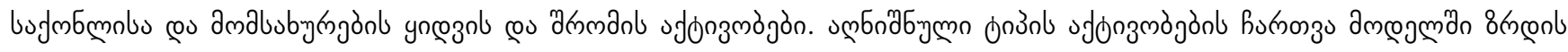

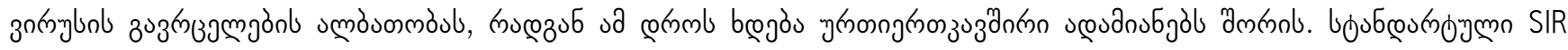

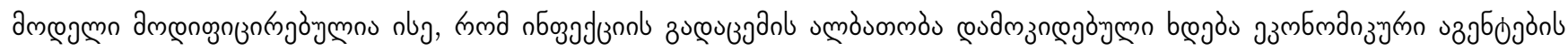

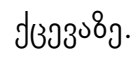

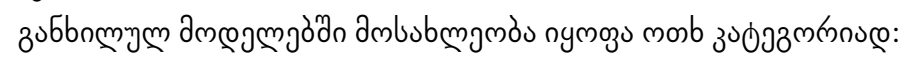

- $S_{t}-\partial_{3}$ (ndbmònotrog (Susceptible),

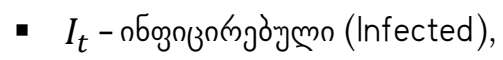

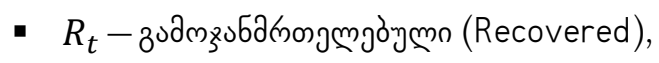

- $D_{t}$ - zungouzzmoña (Deceased). 


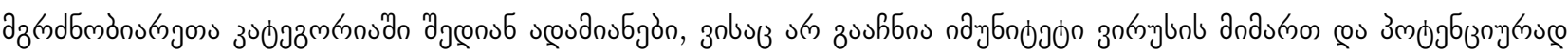

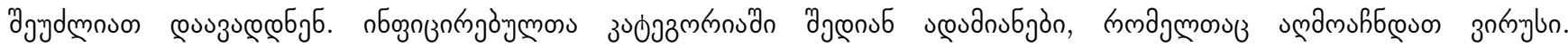

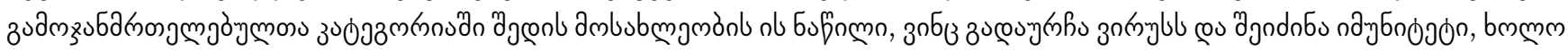

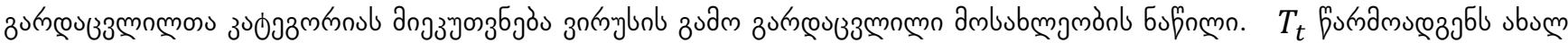

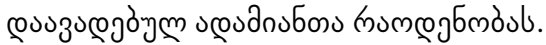

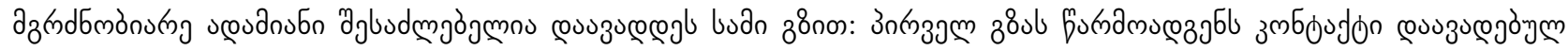

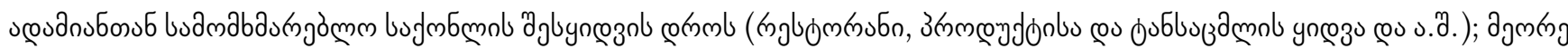

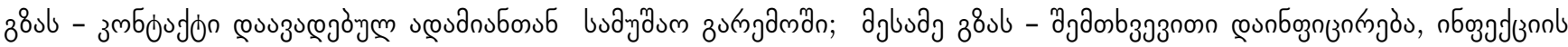

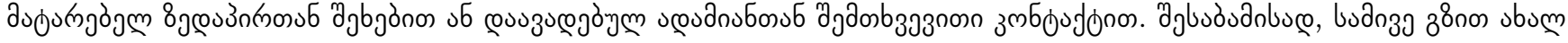

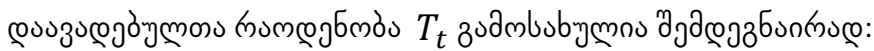

$$
T_{t}=\pi_{1}\left(S_{t} C_{t}^{S}\right)\left(I_{t} C_{t}^{I}\right)+\pi_{2}\left(S_{t} N_{t}^{S}\right)\left(I_{t} N_{t}^{I}\right)+\pi_{3}\left(S_{t} I_{t}^{S}\right),
$$

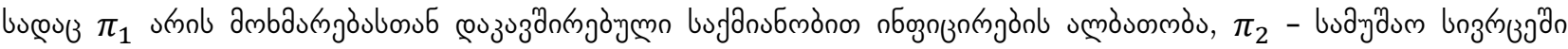

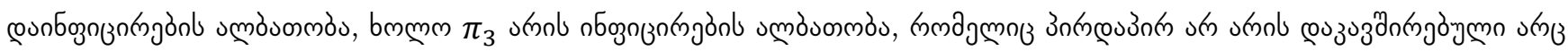

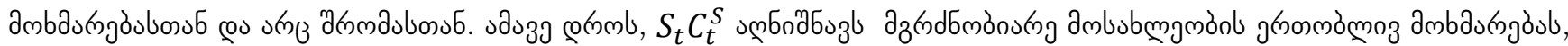

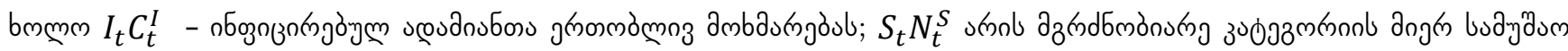

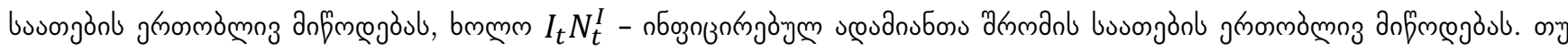

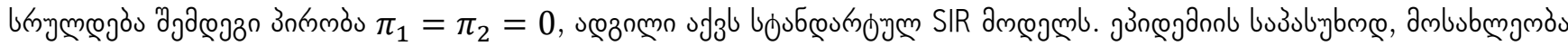

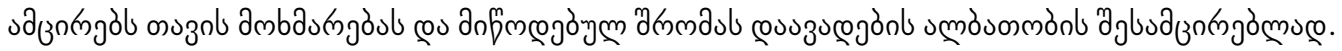

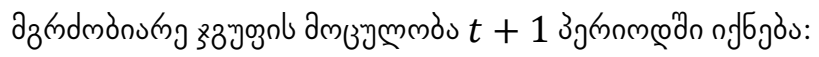

$$
S_{t+1}=S_{t}-T_{t}
$$

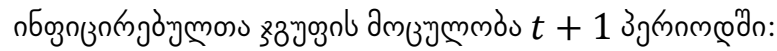

$$
I_{t+1}=I_{t}+T_{t}-\left(\pi_{r}+\pi_{d}\right) I_{t}
$$

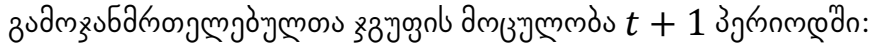

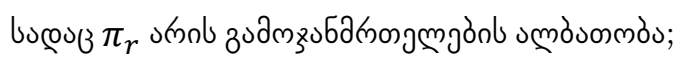

$$
R_{t+1}=R_{t}+\pi_{r} I_{t}
$$

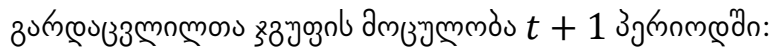

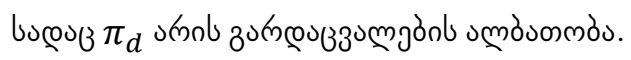

$$
D_{t+1}=D_{t}+\pi_{d} I_{t}
$$

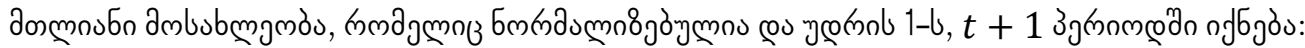

$$
\mathrm{Pop}_{t+1}=\mathrm{Pop}_{t}-\pi_{d} I_{t} \text {. }
$$

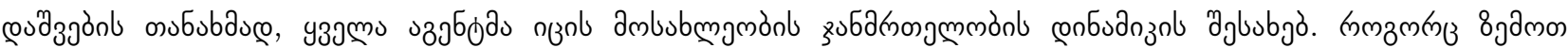

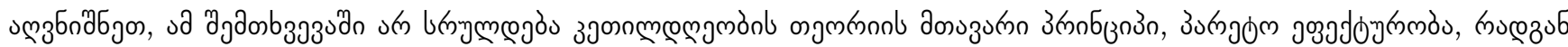

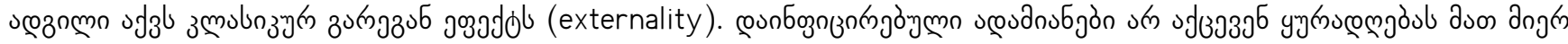

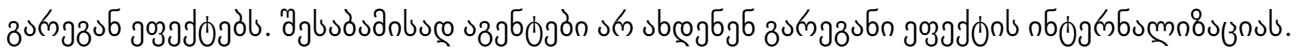

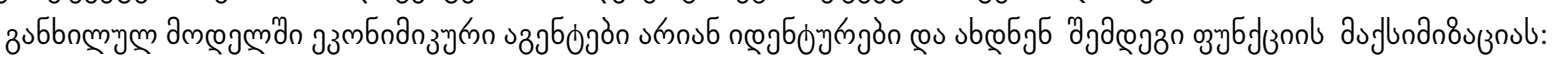

$$
U=\sum_{t=0}^{\infty} \beta^{t} u\left(C_{t}, N_{t}\right),
$$

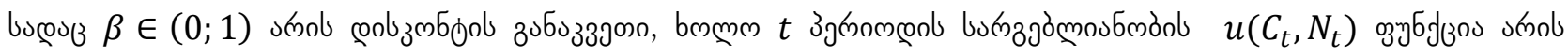

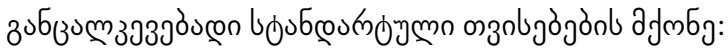

$$
u_{t}\left(C_{t}, N_{t}\right)=\ln C_{t}+\frac{\theta}{2} N_{t}^{2},
$$

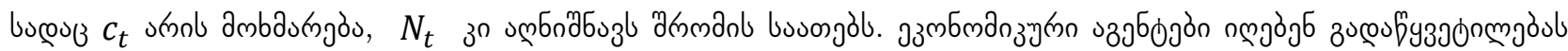

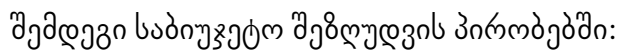

$$
\left(1+\mu_{t}\right) C_{t}=\omega_{t} C_{t}+\Gamma_{t}
$$

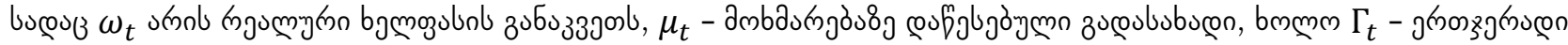

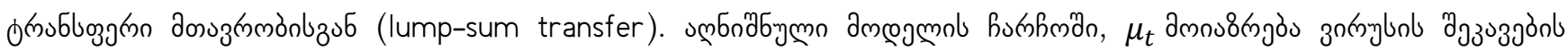




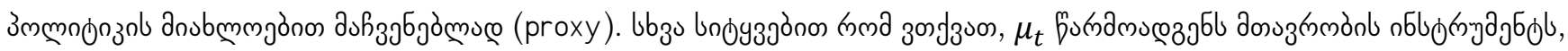
(mnon:

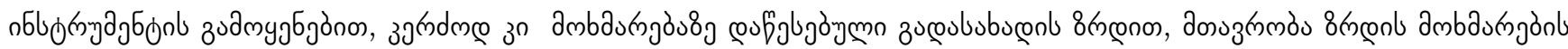

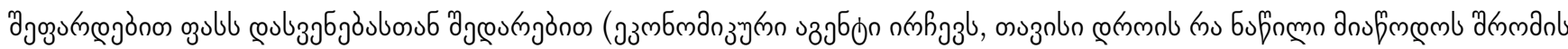

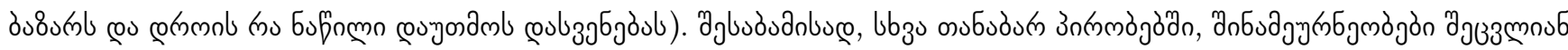

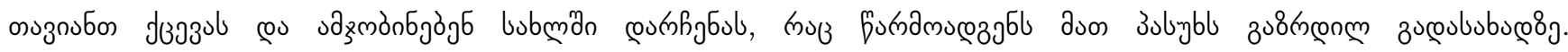

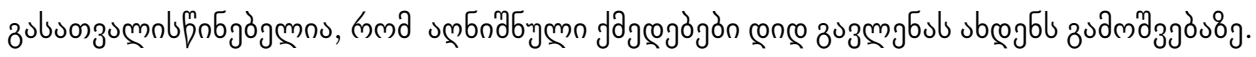

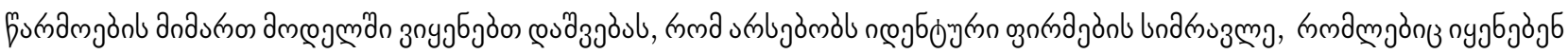

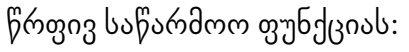

$$
C_{t}=A N_{t}
$$

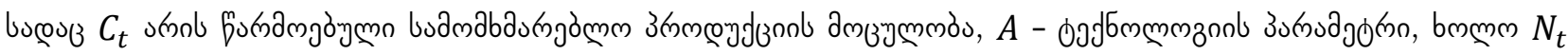

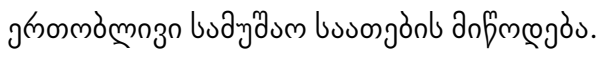

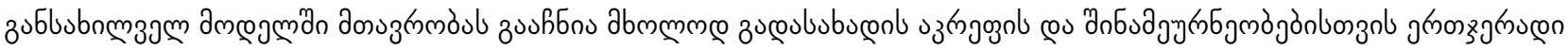

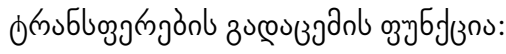

$$
\mu_{t} C_{t}=\Gamma_{t}
$$

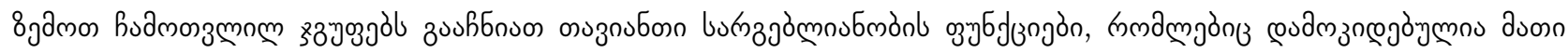

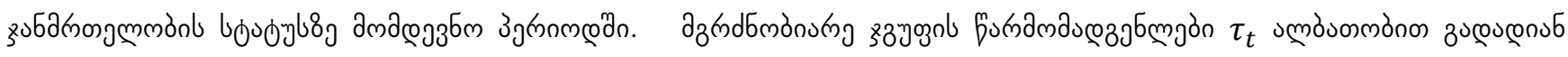

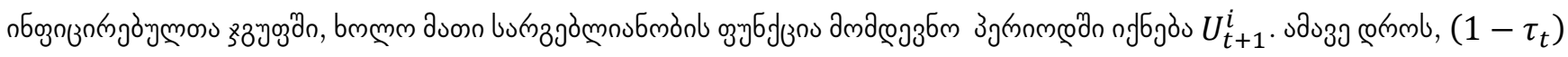

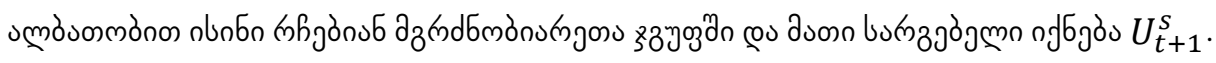

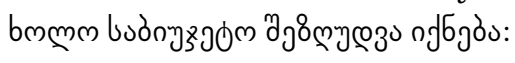

$$
U_{t}^{S}=u\left(C_{t}^{S}, N_{t}^{S}\right)+\beta\left[\left(1-\tau_{t}\right) U_{t+1}^{S}+\tau_{t} U_{t+1}^{i},\right.
$$

$$
\begin{gathered}
\left(1+\mu_{t}\right) C_{t}^{s}=\omega_{t} N_{t}^{s}+\Gamma_{t} \\
\tau_{t}=\pi_{1} C_{t}^{s}\left(I_{t} C_{t}^{I}\right)+\pi_{2} N_{t}^{s}\left(I_{t} N_{t}^{I}\right)+\pi_{3} I_{t} .
\end{gathered}
$$

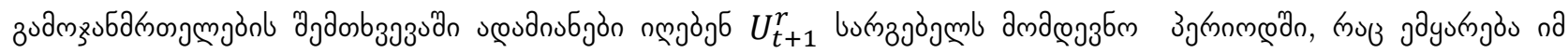

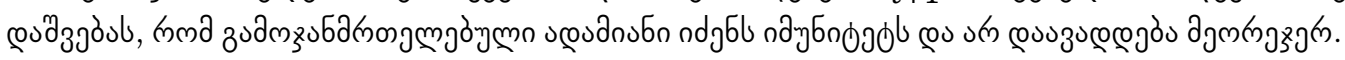

$$
U_{t}^{r}=u\left(C_{t}^{r}, N_{t}^{r}\right)+\beta U_{t+1}^{r} .
$$

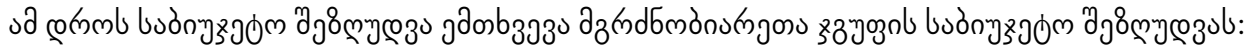

$$
\left(1+\mu_{t}\right) C_{t}^{r}=\omega_{t} N_{t}^{r}+\Gamma_{t} .
$$

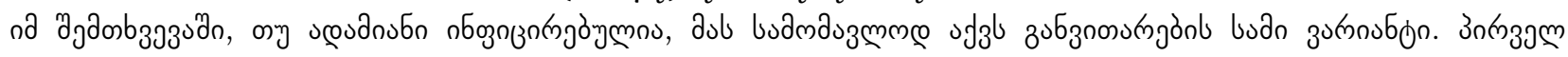

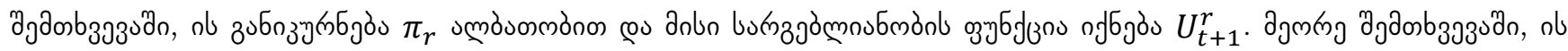

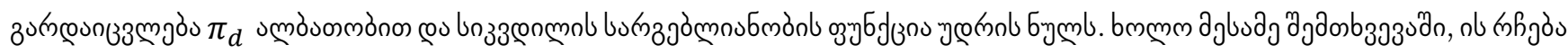

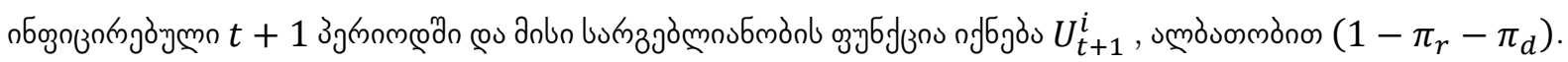

$$
U_{t}^{i}=u\left(C_{t}^{i}, N_{t}^{i}\right)+\beta\left[\left(1-\pi_{r}-\pi_{d}\right) U_{t+1}^{i}+\pi_{r} U_{t+1}^{r}+\pi_{d} 0 .\right.
$$

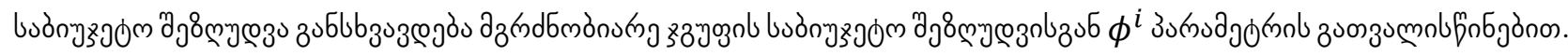

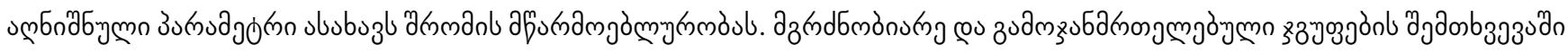
an

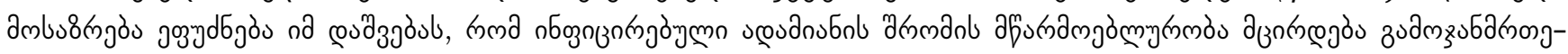

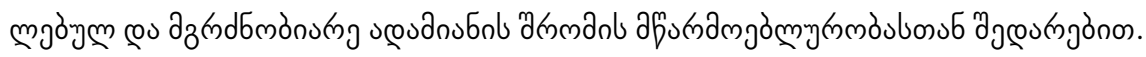

$$
\left(1+\mu_{t}\right) C_{t}^{i}=\omega_{t} \phi^{i} N_{t}^{i}+\Gamma_{t} .
$$

\subsection{3ง $\bmod 00030 \mathrm{~s}$}

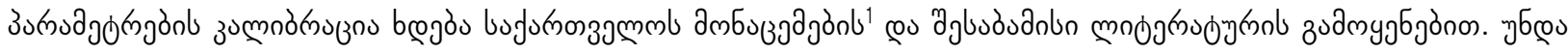

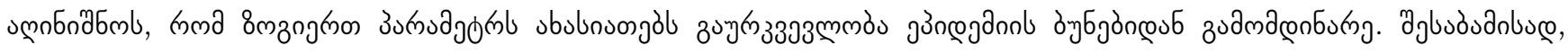

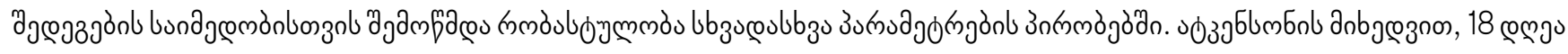




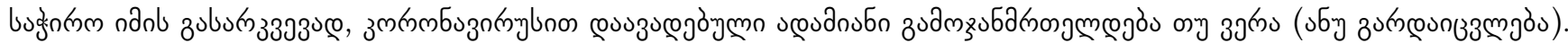

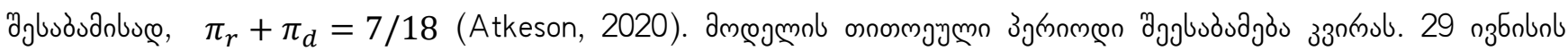

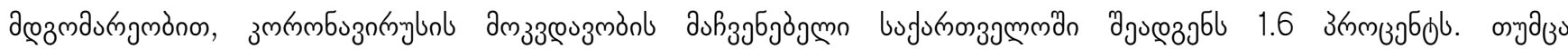

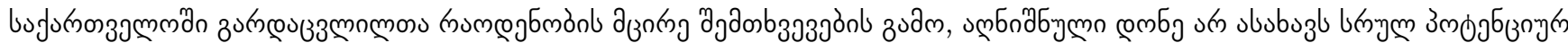

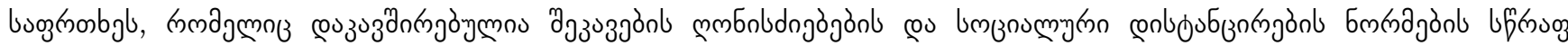

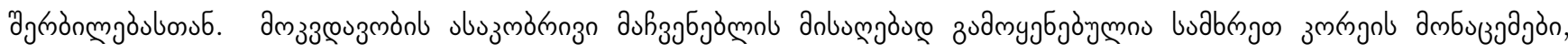

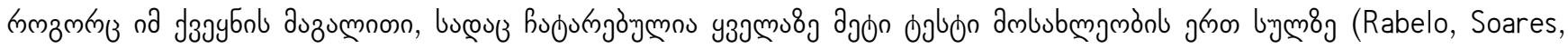

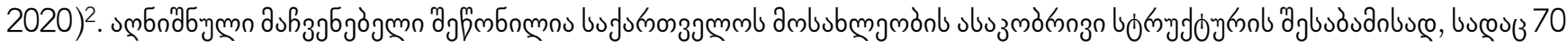

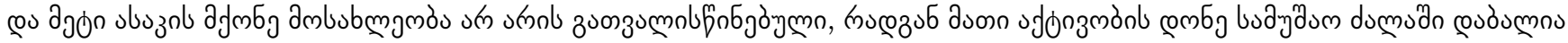

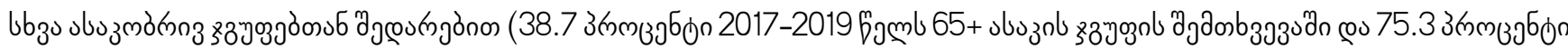

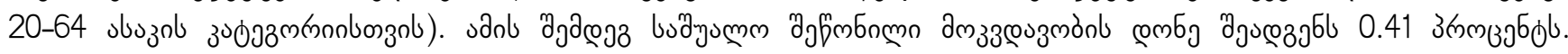

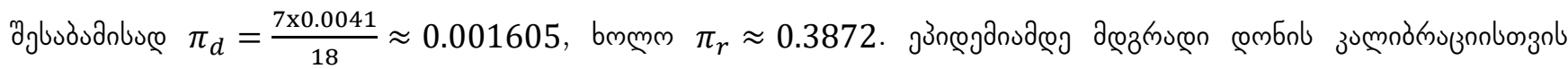

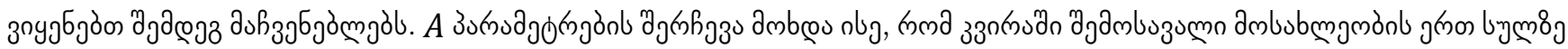

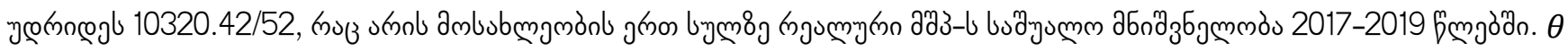

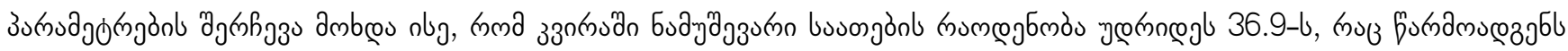

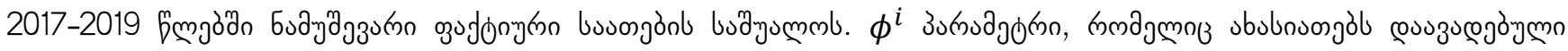

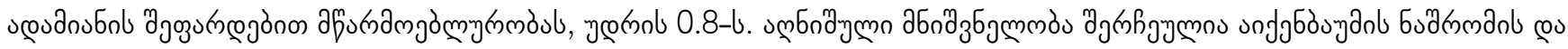

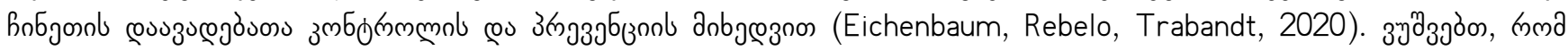

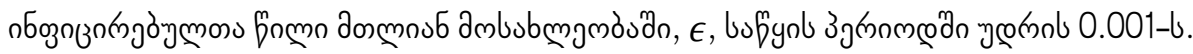

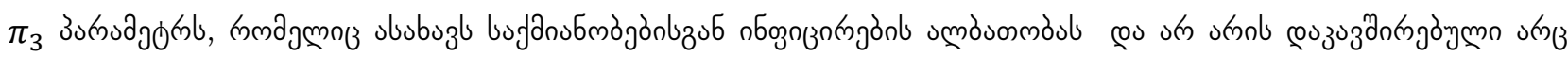

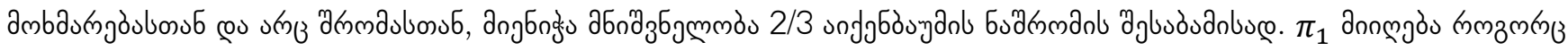

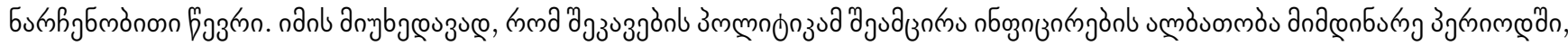

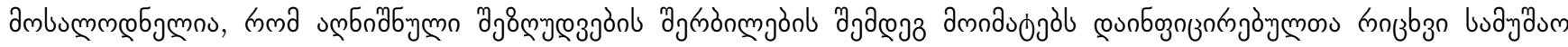

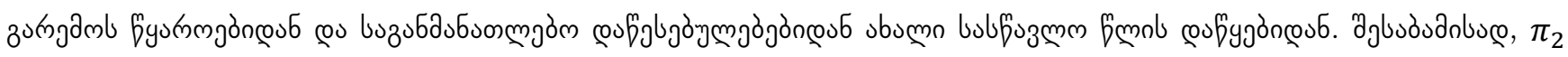

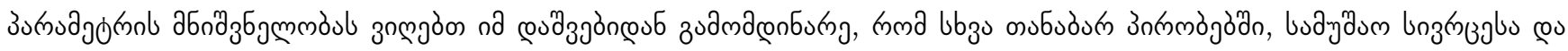

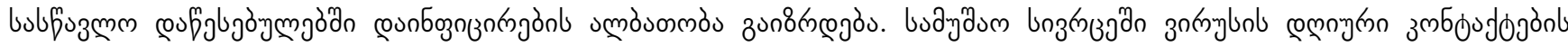

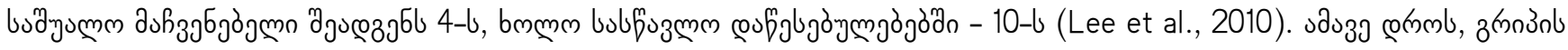

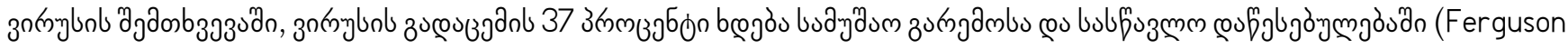

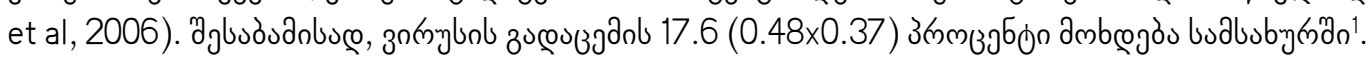

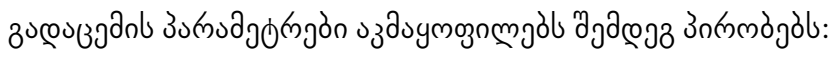

$$
\begin{gathered}
\frac{\pi_{3}}{\pi_{1} C^{2}+\pi_{2} N^{2}+\pi_{3}}=\frac{2}{3} \\
\frac{\pi_{2} N^{2}}{\pi_{1} C^{2}+\pi_{2} N^{2}+\pi_{3}}=0.1764, \\
\frac{\pi_{1} C^{2}}{\pi_{1} C^{2}+\pi_{2} N^{2}+\pi_{3}}=1-\frac{2}{3}-0.1764 .
\end{gathered}
$$

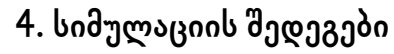

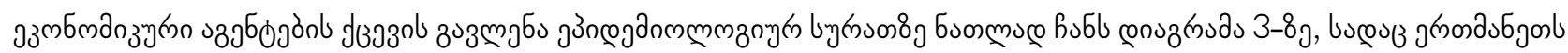

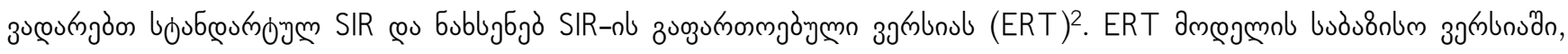

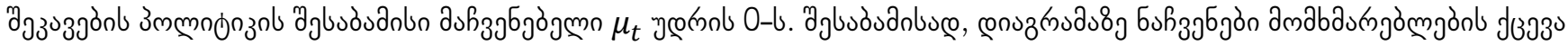

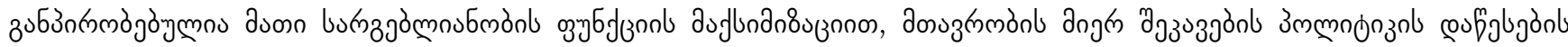

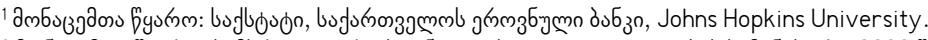

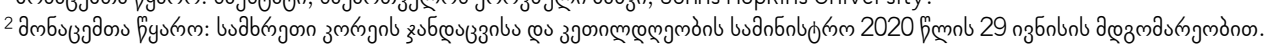




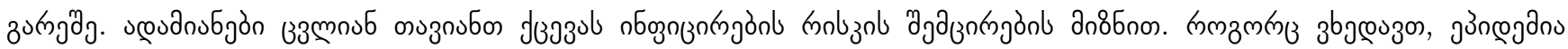

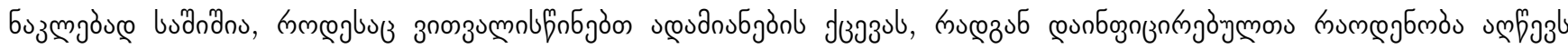

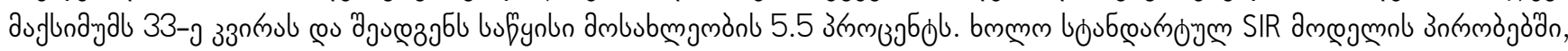

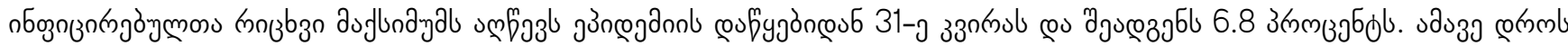

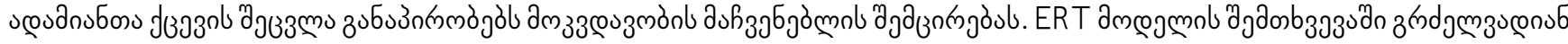

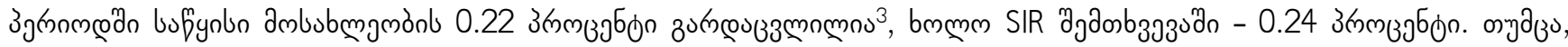

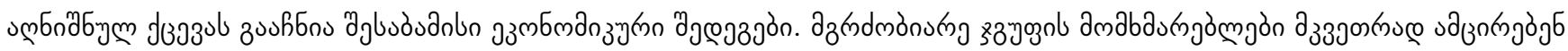

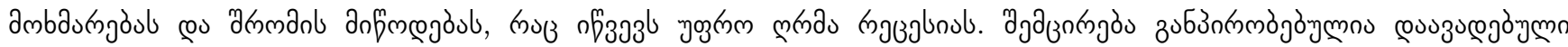

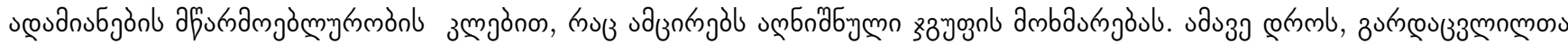

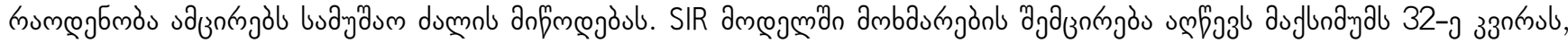

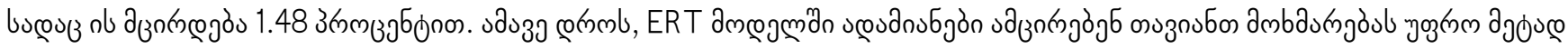

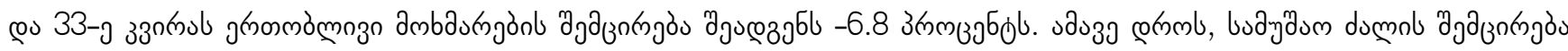

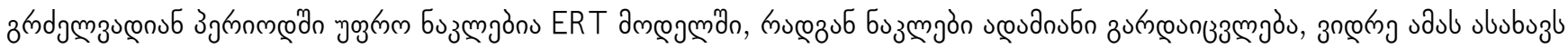
SIR dmegmo.

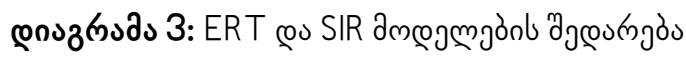
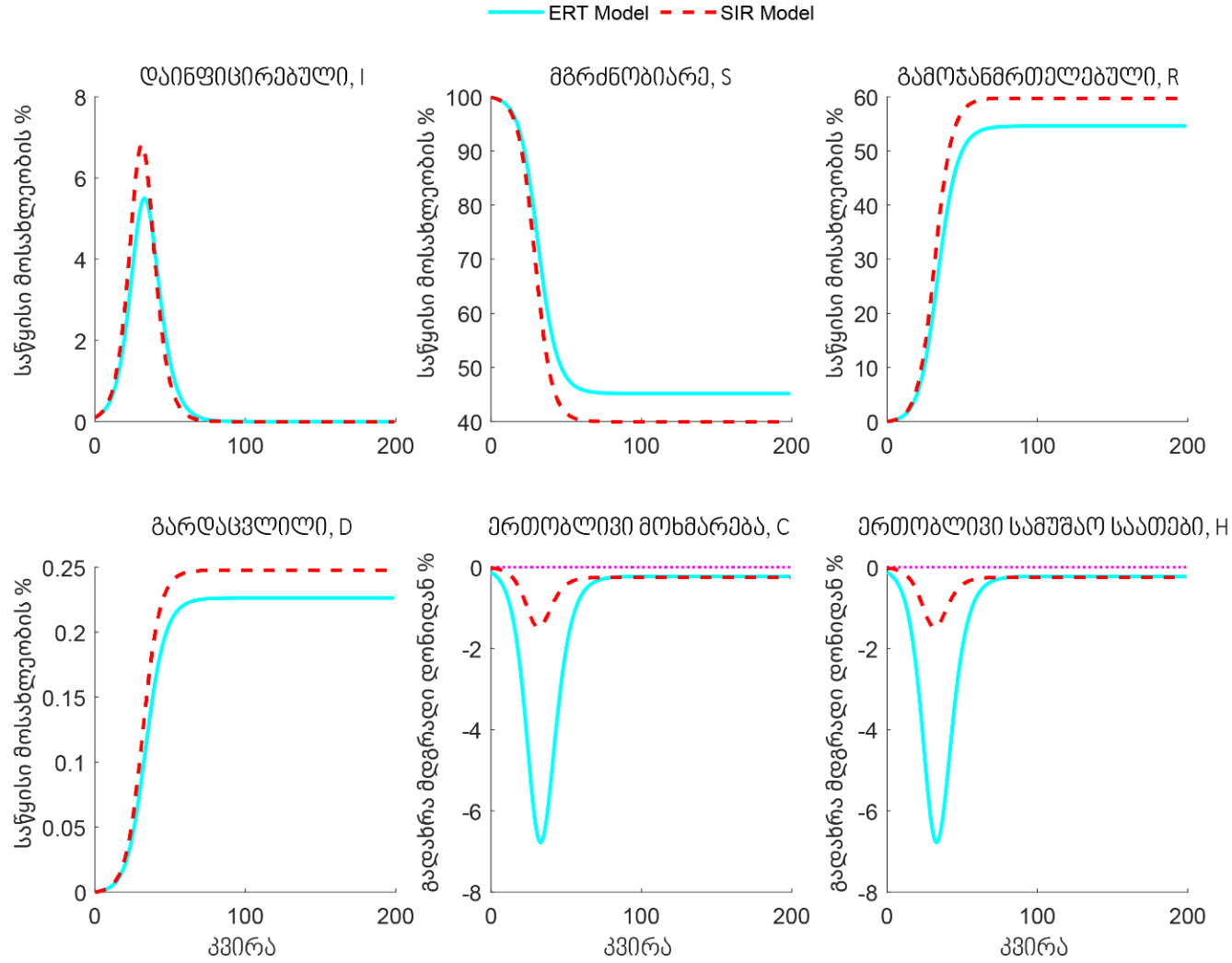

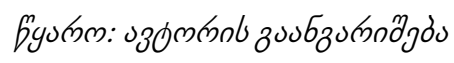

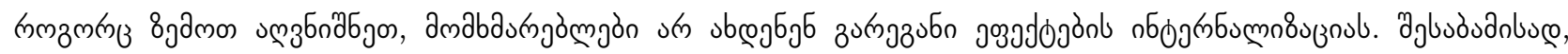

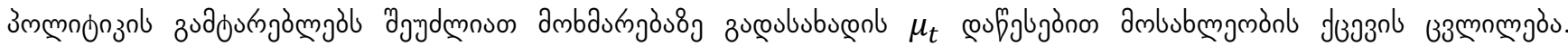

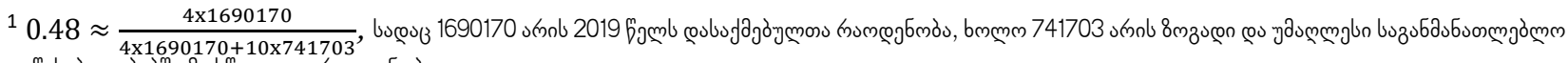

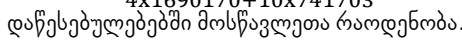

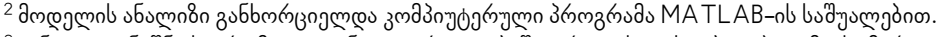

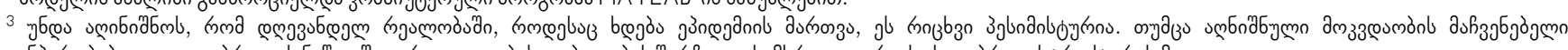

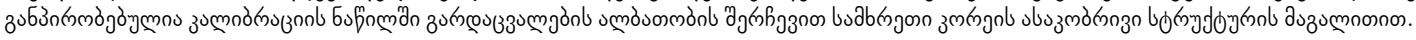




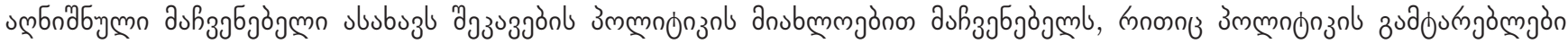

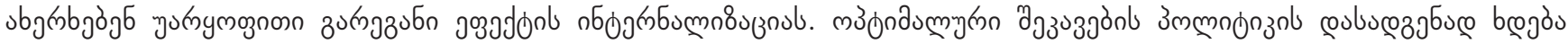

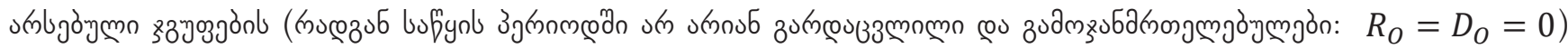
buffynlo z af Trabandt, 2020):

$$
U_{O}=S_{O} U_{O}^{S}+I_{O} U_{O}^{I}
$$

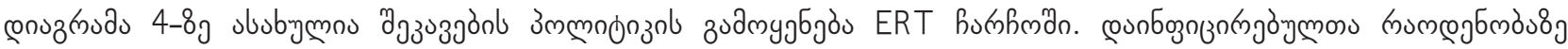

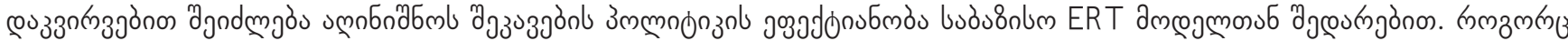

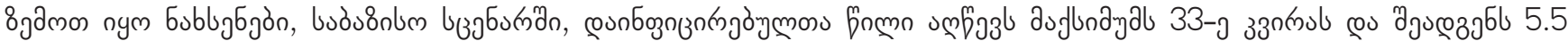

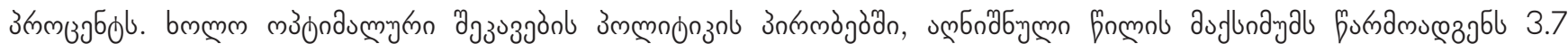

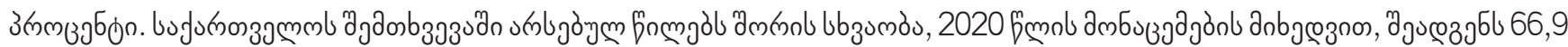

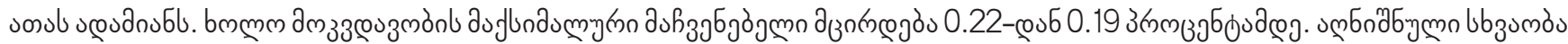

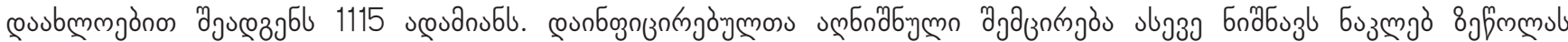

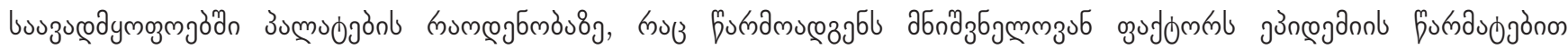

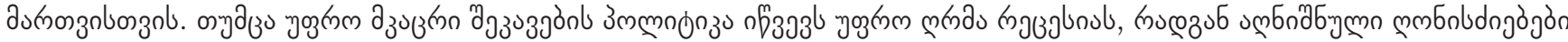

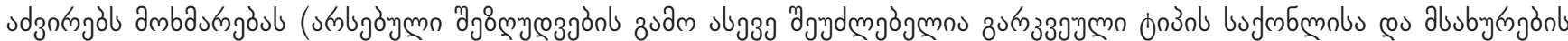

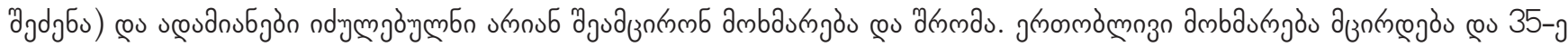

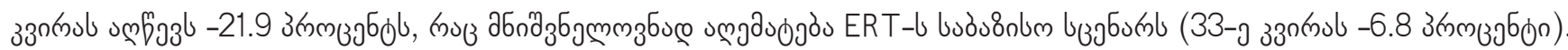

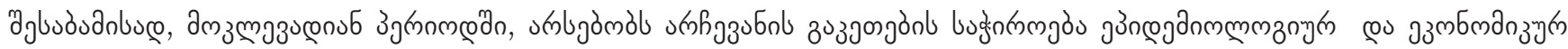
znosurngòjol ammol.

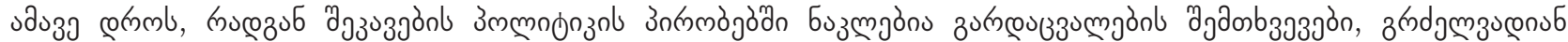

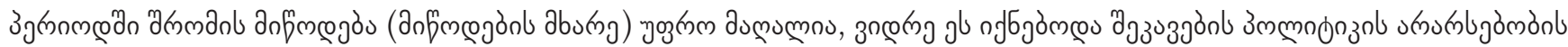

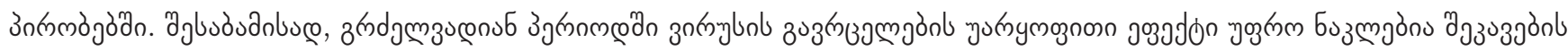

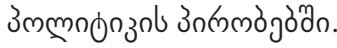

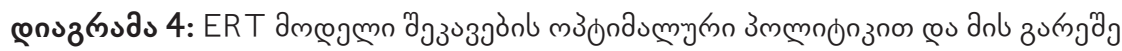

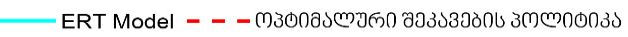
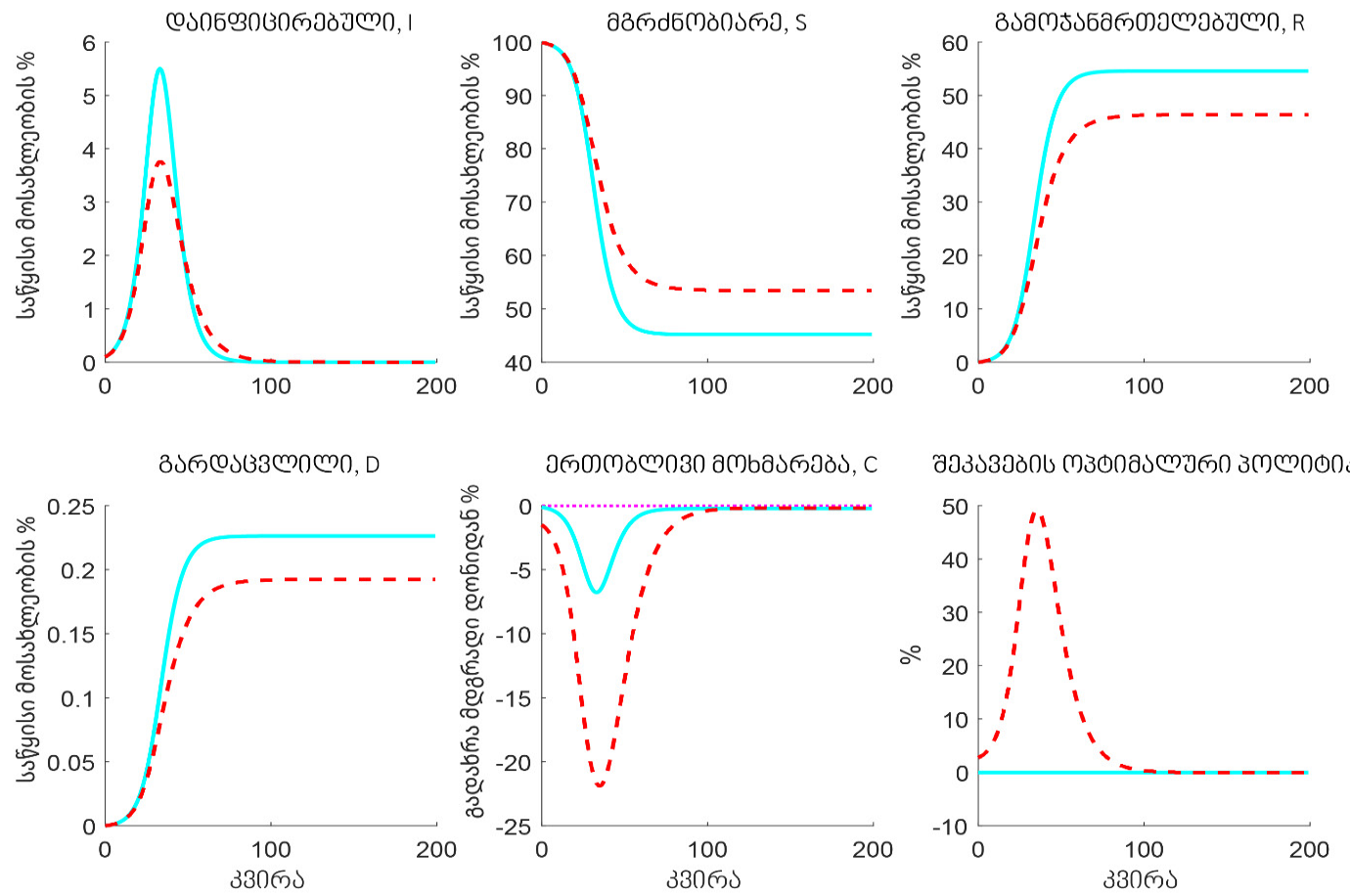

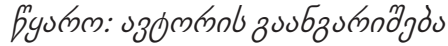




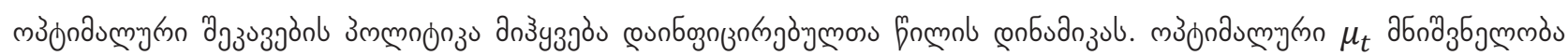

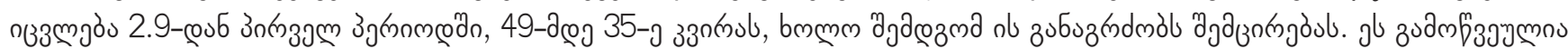

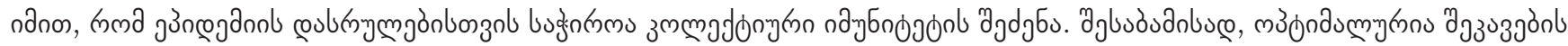

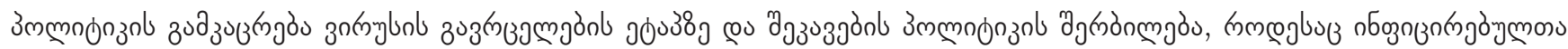

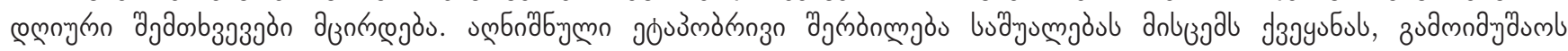

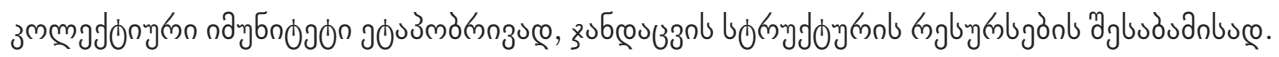

\section{5. $\cos 33^{60}$}

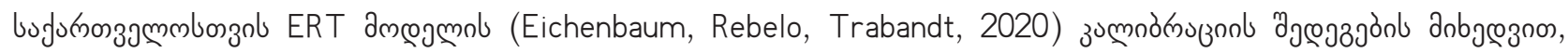

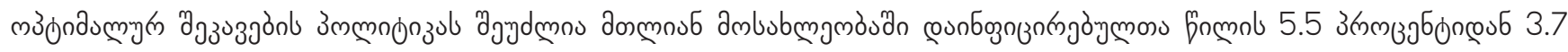

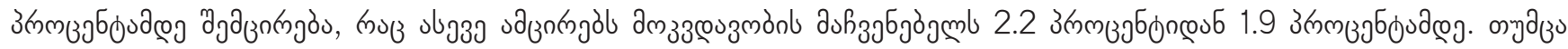

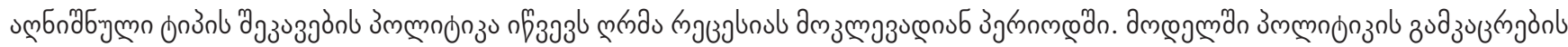

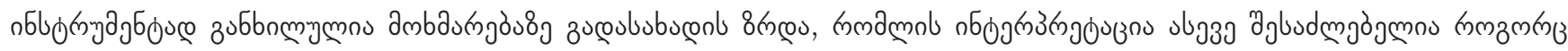

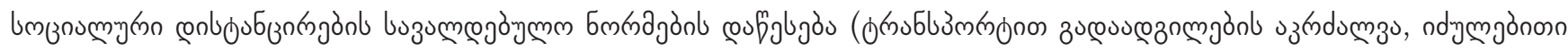

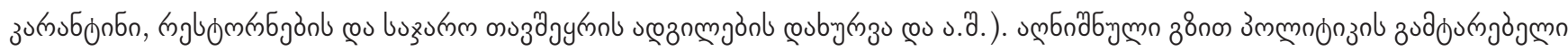

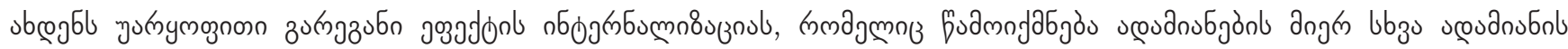

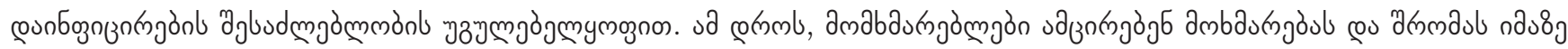

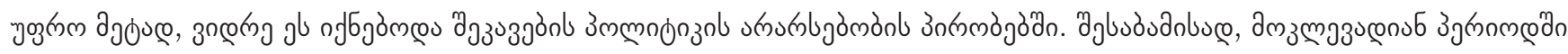

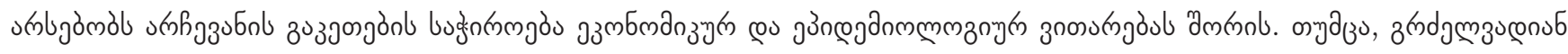

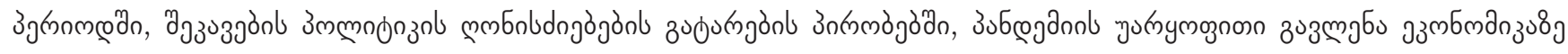

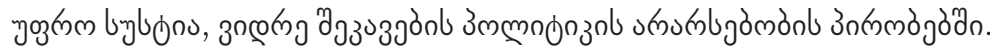

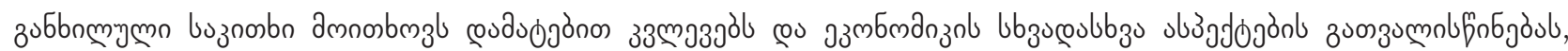

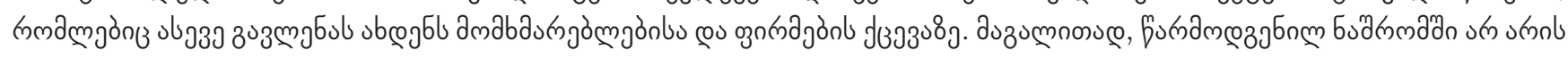

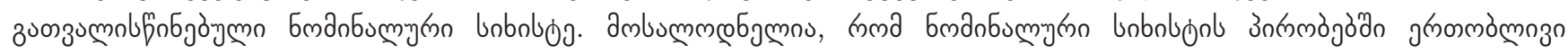

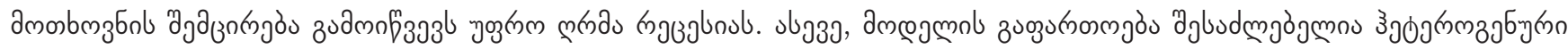

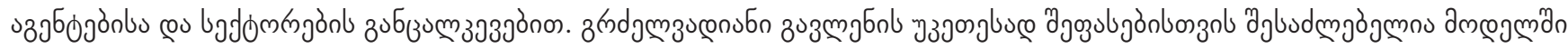

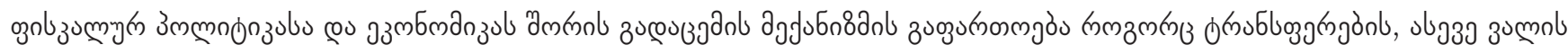

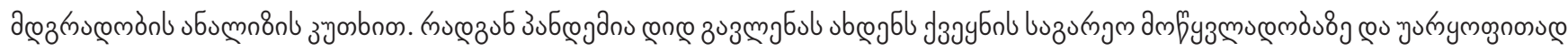

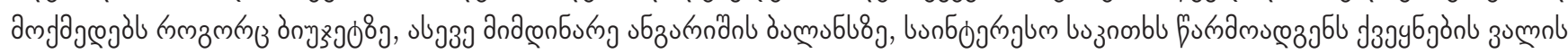

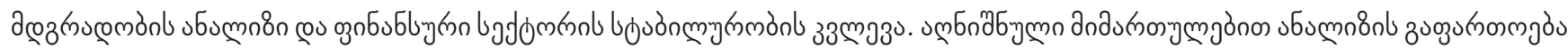

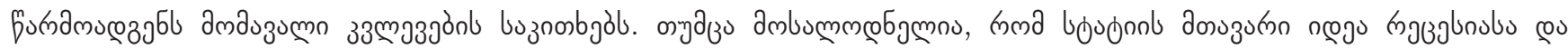

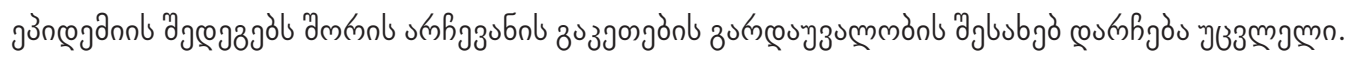

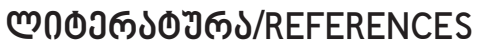

Ananiashvili, I., Gaprindashvili, L. (2020). The Scale of the Spread of COVID -19 in Georgia and Effectiveness of Preventive Measures Implemented by the Government - What Do Models Say? Globalization and Business, 10. (In Georgian).

Atkeson, A. (2020). What will be the economic impact of covid-19 in the us? rough estimates of disease scenarios (w26867). National Bureau of Economic Research.

Barro, R. J., Ursúa, J. F., \& Weng, J. (2020). The coronavirus and the great influenza pandemic: Lessons from the "spanish flu" for the coronavirus's potential effects on mortality and economic activity (w26866). National Bureau of Economic Research.

Eichenbaum, M. S., Rebelo, S., \& Trabandt, M. (2020). The macroeconomics of epidemics (w26882). National Bureau of Economic Research.

Ferguson, N. M., Cummings, D. A., Fraser, C., Cajka, J. C., Cooley, P. C., \& Burke, D. S. (2006). Strategies for mitigating an influenza pandemic. Nature, 442(7101), 448-452.

Georgian Government (2020). Report of conducted measures against COVID-19 by the Georgian Government [COVID-19-is winaamghdeg saqartvelos mtavrobis mier gatarebuli ghonisdziebebis angarishi]. (In Georgian) 
Guerrieri, V., Lorenzoni, G., Straub, L., \& Werning, I. (2020). Macroeconomic Implications of COVID-19: Can Negative Supply Shocks Cause Demand Shortages? (No. w26918). National Bureau of Economic Research.

International Monetary Fund. World Economic Outlook Update: A Crisis Like No Other, An Uncertain Recovery. Washington, DC, June 2020.

International Monetary Fund. World Economic Outlook: The Great Lockdown (2020). Washington, DC, April.

International Monetary Fund. World Economic Outlook Update: Tentative Stabilization, Sluggish Recovery? (2020). Washington, DC, January 2020.

Jibuti, M. (2020). Viral Variations of Political Economy. Round Table "Coronomics". Economics and Business, 2. (In Georgian). Jordà, Ò., Singh, S. R., \& Taylor, A. M. (2020). Longer-run economic consequences of pandemics (w26934). National Bureau of Economic Research.

Kakulia, M. (2020). Lessons from the Coronomic Crisis for Georgia. Round Table "Coronomics". Economics and Business, 2. (In Georgian).

Kermack, W. O., \& McKendrick, A. G. (1927). A contribution to the mathematical theory of epidemics. Proceedings of the royal society of london. Series A, Containing papers of a mathematical and physical character, 115(772), $700-721$.

Kovzanadze, I. (2020). Economic Challenges and Prospects During the COVID-19 Infection. Round Table "Coronomics". Economics and Business, 2. (In Georgian).

Krueger, D., Uhlig, H., \& Xie, T. (2020). Macroeconomic dynamics and reallocation in an epidemic (w27047). National Bureau of Economic Research.

Lee, B. Y., Brown, S. T., Cooley, P. C., Zimmerman, R. K., Wheaton, W. D., Zimmer, S. M., ... \& Burke, D. S. (2010). A computer simulation of employee vaccination to mitigate an influenza epidemic. American journal of preventive medicine, 38(3), 247-257.

Mekvabishvili, E. (2020). The Impact of the Global Coronomic Crisis on the Georgian Economy. Round Table "Coronomics". Economics and Business, 2. (In Georgian).

Papava, V. (2020). Coronomics and Qualitatively New Economic Crisis. Round Table "Coronomics". Economics and Business, 2. (In Georgian).

Rabelo, M., \& Soares, J. (2020). The Macroeconomics of Epidemics: results for Brazil. 